\title{
Study of the Fractal and Multifractal Scaling Intervening in the Description of Fracture Experimental Data Reported by the Classical Work: Nature 308, 721-722(1984)
}

\author{
Liliana Violeta Constantin ${ }^{\mathbf{1}}$ and Dan Alexandru Iordache ${ }^{\mathbf{2}}$ \\ ${ }^{1}$ Physics Faculty, University of Bucharest, P.O. Box MG-11, 077125 Bucharest, Romania \\ 2 Physics Department, University "Politehnica" of Bucharest, Splaiul Independentei, \\ 060042 Bucharest, Romania \\ Correspondence should be addressed to Liliana Violeta Constantin, \\ liliana2009constantin@yahoo.com
}

Received 9 September 2011; Accepted 4 October 2011

Academic Editor: Cristian Toma

Copyright (c) 2012 L. V. Constantin and D. A. Iordache. This is an open access article distributed under the Creative Commons Attribution License, which permits unrestricted use, distribution, and reproduction in any medium, provided the original work is properly cited.

Starting from the experimental data referring to the main parameters of the fracture surfaces of some 300-grade maraging steel reported by the classical work published in Nature 308, 721722(1984), this work studied (a) the multifractal scaling by the main parameters of the slit islands of fracture surfaces produced by a uniaxial tensile loading and (b) the dependence of the impact energy to fracture and of the fractal dimensional increment on the temperature of the studied steels heat treatment, for the fracture surfaces produced by Charpy impact. The obtained results were analyzed, pointing out the spectral (size) distribution of the found slit islands in the frame of some specific clusters (fractal components of the multifractal scaling) of representative points of the logarithms of the slit islands areas and perimeters, respectively.

\section{Introduction: Complexity, Universality, Power Laws, and Fractal Scaling}

As it is well-known, one of the most important present topics refers to the obtainment of scientific information about the complex materials and systems [1-3].

The main founders of the complexity theory in physics have pointed out [4-7] (see also the synthesis review $[8,9]$ ) that several completely different complex systems (computer arrays, complex random (Internet, particularly), robots, networks, social sciences, biology (with some specific topics: colonies, swarms, immunology, brain, genetics, and proteomics), 
economics, mathematics, glasses, agents, and cognition [10-13], etc.) have some common features centered on their statistical behavior and the corresponding phase transforms $[4,5,8,9]$ and chemical reactions, particularly, as well as of some dynamic aspects [14-16], nonlinear effects [17], and so forth. It results that these complex systems have certain universality properties, which—due to their generality (see, e.g., [8]) — can be described only by some specific numbers (the so-called similitude numbers, or criteria [18-20]).

How could it be possible to describe dimensional (physical, particularly) quantities only by numbers? The answer is obtained from the examination of: (a) predictions of Anderson $[4,5,8,9]$ relative to the "explosive" autocatalytic (exponential) growth following the spontaneous symmetry breaking inside the specific complex systems (one finds that a certain dimensional parameter $p$ has to be described by its logarithm: $\ln p$ ), (b) Dalton's law of "defined proportions", intervening in the theory of chemical reactions (somewhat similar to the phase transforms) [3]: $d \xi=-d v_{1} / v_{1}=-d v_{2} / v_{2}=\cdots=+d v_{N} / v_{N}$, where the sign " -" corresponds to substances that disappear during the considered chemical reaction, while the sign " + " corresponds to the appearing substances, finding that the degree of advance $\xi$ of the considered reaction can be expressed by means of $\ln v_{j}$, where $v_{j}$ is the amount (e.g., number of moles) of one of the substances participating in the chemical reaction, (c) statistical expression of the thermodynamic entropy (describing the dissipative processes), given by the Planck-Boltzmann's expression: $S=-k \cdot \ln \wp$, where $k$ is the the Boltzmann's constant, where $\wp$ is the probability density, (d) Claude Shannon's expression [21-23] of the individual information quantity: $\mathfrak{I}=-a \cdot \ln \wp(a=$ constant).

The simplest expression (the zero-order approximation) of the relation between a test physical parameter $t(u)$ and the uniqueness one $u$ is, of course, the linear expression:

$$
\ln t=\ln t_{1}+s \cdot \ln u \text {, equivalent to the power law: } t(u)=t_{1} \cdot u^{s} \text {. }
$$

If the uniqueness parameter $u$ corresponds to the size $L$ of the considered complex system, then the power law (1.1) particularizes into the fractal scaling

$$
t(L)=t_{1} \cdot L^{s}
$$

When the relation $\ln t=f(\ln L)$ is more intricate than the linear one, presenting, for example, a certain curvature, then the existing experimental data can be divided in some groups of pairs $\left\{t_{k 1}, L_{k 1} ; \ldots t_{k n}, L_{k n}\right\}$ so that for each group, a specific linear relation is valid: $\ln t_{k i}=$ $\ln t_{1 k}+s_{k} \ln u_{k i}$, equivalent to the fractal scaling: $t_{k i}=t_{1 k} \cdot u_{k i}^{s_{k}}$. Because the prepower coefficient $t_{1 k}$ and the power exponent $s_{k}$ depend on the group $k$ of chosen data, it results that the set of relations $\left\{t_{k i}=t_{1 k} \cdot u_{k i}^{s_{k}} \mid k=1, N\right\}$ corresponds to a multifractal scaling [24, 25].

Some additional detailed studies of the different types of fractal and multifractal scaling were accomplished in the frame of works [26-28].

\section{Critical Findings Referring to the Work Nature, 308, 721-722(1984)}

In 1984, Mandelbrot et al. [29] claimed that the fracture surfaces of metals are fractal (selfsimilar) over a wide range of sizes, and introduced the experimental methods named "slit island analysis" (SIA) and "fracture profile analysis" (FPA). As the large majority of papers published by Nature (average impact factor 12.86 in 1985 and 24.82 in 1996), the aboveindicated work had a high scientific impact: we identified [30, 31] at least 26 papers and 
books published only in the following 10 years (up to 1993, inclusively [30]), studying the fractal character of the fracture surfaces. Despite of its large impact, the hypothesis of Mandelbrot et al. [29] was somewhat restricted by the following studies: (a) even the papers of Underwood [32], Pande et al. [33], Lung and Mu [34], and Huang et al. [35] affirmed that the fracture surfaces of metals can be approximately considered to possess a certain fractal character, (b) Underwood and Banerji [32] concluded that the slit island analysis itself was imperfect in nature as a method for measuring the fractal dimension of fractured surfaces, (c) Lung and $\mathrm{Mu}$ [34] found that the fractal dimension was largely affected by the measuring ruler employed and postulated the concept of inherent measuring ruler, (d) Huang et al. [35] pointed out that how to determine the fractal dimension of a fractured surface has always been a problem of "argument", and (e) Williford [36] tried to explain the obtained results in terms of multifractals, but this explanation seemed not to be satisfactory for some experimental results $[37,38]$, and so forth.

The detailed numerical analysis accomplished in the frame of this work pointed out that the main missing elements of work [29] are the following:

(a) no justification of the indicated values of fractal dimensional increment from the capture of Figure 1 [29],

(b) no analysis of the multifractal scaling of the $\log A=f(\log P)$ dependence corresponding to the slit islands areas and perimeters, respectively,

(c) the regression line: impact energy $=f$ (fractal dimensional increment) from Figure 3 [29] is obviously inexact, and it does not consider the corresponding possible nonlinear dependence,

(d) the dependence of the fractal dimensional increment on the temperature of the heattreatment of the 300-grade maraging steel Charpy impact specimens studied by Figure 3 [29] was not studied.

\section{Procedure Intended to the Evaluation of the Fractal Dimension of the Slit Islands}

In order to evaluate the slope of the regression line $\log A=f(\log P)$, the numerical values of the decimal $\log$ arithms $\log A, \log P$ of the slit islands areas and perimeters, respectively, (indicated by Figure 1 [29]) were firstly evaluated by means of the scanning procedure [39]. We obtained $s \equiv D^{\prime} \cong 1.6225=D-1=i_{F}$, in considerable disagreement with the values 1.28 and 1.26 indicated by the capture of Figure 1 [29].

Starting from the interpretation provided by the monograph [40, pages 64-65] of the experimental data obtained by means of the slit island method, according to whom (a) the cross-section of area $A$ of the fractured material is not fractal; therefore, this area is proportional to the square of the slit island average radius $R: A \propto R^{2}$, while (b) the perimeter $P$ of the slit island is really fractal (of dimension $D-1$, where $D$ is the dimension of the fracture surface); therefore, $P \propto R^{D-1}$, and we have found that $A \propto P^{2 /(D-1)}$ and the slope of the $\log A=f(\log P)$ plot is: $s=2 /(D-1)$. From this relation, we obtained, in good quantitative agreement with the indicated fractal dimensional increment, $i_{F}=D-1$ values indicated in the caption of Figure 1 [29] as well as with the results obtained by other similar works (e.g., [41]). 
Table 1: Main features of the fractal: $\log A=c_{0}+c_{1} \log P$ and multifractal (parabolic): $\log A=c_{o}+c_{1} \log P+$ $c_{2}(\log P)^{2}$ scalings of the parameters $A, P$ of the slit islands of fracture surfaces reported by Figure 1 [29].

\begin{tabular}{lcc}
\hline $\begin{array}{l}\text { The type of the } \log A=f(\log P) \text { correlation } \\
\text { (scaling) }\end{array}$ & $\begin{array}{c}\text { Regression line } \\
\text { (fractal scaling) }\end{array}$ & $\begin{array}{c}\text { Parabolic correlation } \\
\text { (multifractal scaling) }\end{array}$ \\
\hline$c_{0}$ & -1.776 & -1.5686 \\
$c_{1}$ & 1.6265 (average slope) & +2.39074 \\
$c_{2}$ & 0 & -0.16965 \\
Correlation coefficient & 0.9655 & 0.9792 \\
$\begin{array}{l}\text { Average relative error (\%) for all 41 studied slit } \\
\text { islands }\end{array}$ & $7.540 \%$ & $7.346 \%$ \\
$\begin{array}{l}\text { Average relative error for the 6 extreme (first 3 } \\
\text { and last 3) representative points of Figure 1 }\end{array}$ & $10.134 \%$ & $8.733 \%$ \\
{$[24,25]$} & & $2.4487 \cdots 3.0514$ \\
$\begin{array}{l}\text { Apparent fracture surface fractal dimension: } \\
D_{M}=1+\text { slope }\end{array}$ & 2.6265 & $1.975 \cdots 2.380$ \\
$\begin{array}{l}\text { Fracture surface fractal dimension according to } \\
\text { our considerations (this work): } D_{s}=1+2 / \text { slope }\end{array}$ & 2.23 & \\
\hline
\end{tabular}

\section{Study of the Multifractal Scaling of the $\log A=f(\log P)$ Dependence}

Taking into account that all 6 extreme (first 3 and last 3) representative points of Figure 1 [29] are located under the regression line, we assumed that a nonlinear (even a parabolic) $\log A=f(\log P)$ expression could agree better with the experimental data reported by this figure. To check this assumption, we used the procedures of the well-known classical gradient method [42-44] in order to find the parameters of the parabolic correlation

$$
\log A=c_{2}(\log P)^{2}+c_{1} \log P+c_{0}
$$

which ensure the best fit of the experimental data of Figure 1 [29].

The obtained results are synthesized by Table 1 .

One finds that the explanation given by Williford [36], in terms of multifractals, of the experimental data referring to the fracture surfaces is more realistic than the initial Mandelbrot's hypothesis. We have to underline that this explanation (multifractals) is supported also by the results obtained by Carpinteri and Chiaia $[24,25]$ especially for concrete samples.

The new versions of Figures 1 and 3 [29], after our numerical conversion (using the method of work [39]) of the experimental data indicated by these figures and the following parabolic fit (for the $\log A=f(\log P$ ) pairs), and the least-squares fit (for the fractal dimensional increment $=f($ impact energy)) are presented below in the frame of our Figures 1 and 2 .

\section{Towards the Fractal Components of the Multifractal Set of Fracture Surfaces Slit Islands of the Maraging Steels Studied by [29]}

Taking into account the practical continuous change of the slope of the $\log A=f(\log P)$ plot, the definition of the fractal components of the multifractal set of fracture surfaces slit islands is strongly related to the experimental accuracy of the $\log A, \log P$ parameters. As the accuracy of these parameters is not known, a certain image on these fractal components 


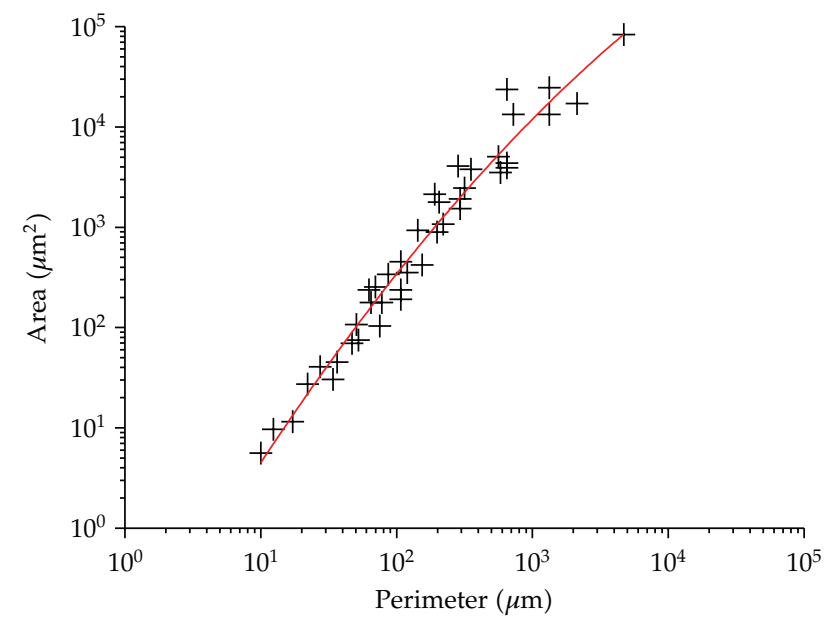

Figure 1: The new (improved) version of Figure 1 [29] after [29], the numerical conversion (using method [39]) of the corresponding experimental data and the parabolic fit of the $\log A=f(\log P)$ pairs.

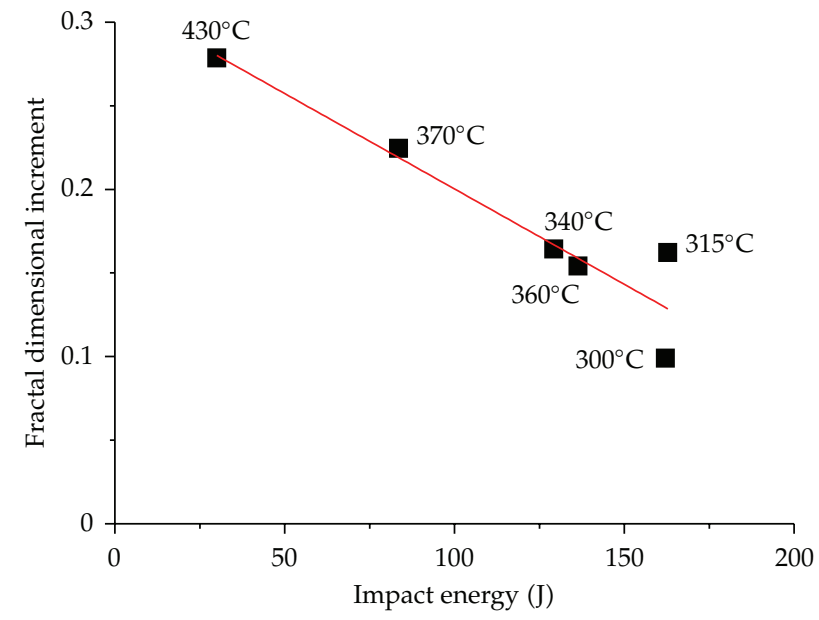

Figure 2: The new (corrected) version of Figure 3 [29] after the numerical conversion (using the method [39]) of the corresponding experimental data, and the least-squares fit of the fractal dimensional increment $=f$ (impact energy) dependence data.

can be obtained starting from the identification of clusters of representative points $\log A$, $\log P$.

We defined the $\log A, \log P$ clusters starting from the distances between the nearest representative points in the space $\log A, \log P$. If the distance between the nearest representative points belonging to 2 neighbor sets is considerably larger than that for the nearest such points belonging to each set, these neighbor sets correspond to the desired clusters.

Using this procedure, we have identified 6 clusters in the $\log A, \log P$ space of Figure 1 [29], defined by the pairs of $\log A, \log P$ coordinates corresponding to the marginal representative points of each cluster.

These clusters of representative points in the $\log A, \log P$ space are gathered around some average $(\log P)_{i}$ values $(i=1, N)$. For each cluster of representative points, the local 
Table 2: The "spectral" (size) distribution of the clusters of representative points in the $\log A, \log P$ plot involved by Figure 1 [26-29] as representing the fractal components of the multifractal scaling of the $\log A=f(\log P)$ dependence.

\begin{tabular}{|c|c|c|c|c|c|c|}
\hline $\begin{array}{l}\text { Interval of fractal } \\
\text { increment values }\end{array}$ & $(0.975 ; 1.014)$ & $(1.034 ; 1.140)$ & $(1.123 ; 1.213)$ & $(1.205 ; 1.297)$ & $(1.283 ; 1.392)$ & $(1.392 ; 1.747)$ \\
\hline $\begin{array}{l}\text { Pairs of values of } \\
\text { the slit islands } P \\
\text { Perimeter }(\mu \mathrm{m}) \\
\text { and area }\left(\mu \mathrm{m}^{2}\right) \\
\end{array}$ & $\begin{array}{c}(10.00 ; 5.62) \\
\ldots \\
(17.15 ; 11.55)\end{array}$ & $\begin{array}{c}(22.07 ; 27.38) \\
\ldots \\
(74.99 ; 103.7)\end{array}$ & $\begin{array}{c}(62.64 ; 237.1) \\
\ldots \\
(154.0 ; 421.7)\end{array}$ & $\begin{array}{c}(143.3 ; 930.6) \\
\ldots \\
(316.2 ; 2458)\end{array}$ & $\begin{array}{c}(283.9 ; 4068) \\
\ldots \\
(649.4 ; 4371)\end{array}$ & $\begin{array}{c}(649 . ; 23714) \\
\ldots \\
(4698 ; 83536)\end{array}$ \\
\hline $\begin{array}{l}\text { Number of } \\
\text { representative } \\
\text { points in Figure } 1 \\
{[1-3]}\end{array}$ & 3 & 8 & 10 & 8 & 6 & 6 \\
\hline $\begin{array}{l}\text { Percentage of } \\
\text { representative } \\
\text { points }\end{array}$ & $7.318 \%$ & $19.512 \%$ & $24.390 \%$ & $19.512 \%$ & $14.634 \%$ & $14.634 \%$ \\
\hline
\end{tabular}

slope $s_{i}=2 c_{2}(\log P)_{i}+c_{1}$ of the multifractal scaling $\log A=c_{2}(\log P)^{2}+c_{1} \log P+c_{0}$ and the local fractal dimensional increment $i_{F i}=2 / s_{i}$ were evaluated, the obtained results being synthesized by Table 2 . The synthesis of these clusters features as well as the corresponding fractal dimensions (or increments) corresponding to each cluster (as a specific representative of the fractal components of the multifractal set of fracture surfaces slit islands) is presented by Table 2 .

One finds that the small values of the fractal dimension correspond to slit islands of relatively small dimensions (perimeters of the magnitude order of $\mu \mathrm{m}$ ), corresponding to fracture surfaces not too curly, and even involving some surface breaks (which could explain eventually the seldom values little less than 2 of the fractal dimension corresponding to some small parts of the fracture surface).

\section{Study of the Fractal Dimensional Increment of the Fracture Surfaces Produced by Impact on the Temperature of the Steels Heat Treatment}

Unlike the fracture surfaces produced by uniaxial tensile loading, whose characteristic parameters were reported for the 300-grade maraging steel by Figure 1 [29], the last part of this work (Figure 3 [29]) reports the main features of the fracture surfaces produced by impact.

The evaluation of the slope $s$ and intercept $i$ of the regression line $E_{\text {imp }}(\mathrm{J})=s \cdot t_{\text {heat }}+i$ describing the impact energy to fracture in terms of the temperature of the studied steels heat treatment led us to the results: $s \cong-1.069 \mathrm{~J} /{ }^{\circ} \mathrm{C}, i \cong 494.21 \mathrm{~J}$ with a correlation coefficient $r \cong-0.9563$ and a square mean relative error of $10.05 \%$.

Similarly, the evaluation of the slope $s^{\prime}$ and of the intercept $i^{\prime}$ of the regression line $i_{F}=s^{\prime} \cdot t_{\text {heat }}+i^{\prime}$ describing the fractal dimensional increment of the fracture surface produced by impact in terms of the temperature of the studied steel heat treatment leads to the results $s^{\prime} \cong 1.25 \cdot 10^{-3}\left({ }^{\circ} \mathrm{C}\right)^{-1}, i^{\prime} \cong-0.260$, with a correlation coefficient $r^{\prime} \cong 0.9243$ and a square mean relative error of $10.971 \%$.

One finds that, as it was expected, (a) the impact energy to fracture decreases (approximately linearly, up to $450^{\circ} \mathrm{C}$ ) with the temperature of the studied steels heat treatment and 
(b) the fracture surface deformation (from its ideal planar shape), measured by its fractal dimensional increment, increases with the temperature of the heat treatment.

It was possible to obtain also the parameters of a more exact (than that performed in the frame of Figure 3 [26-29]) regression line $E_{\text {imp }}(J)=s^{\prime \prime} \cdot i_{F}+i^{\prime \prime}$ describing the dependence of the impact energy to fracture on the corresponding fracture surface deformation (fractal dimensional increment) $s^{\prime \prime} \cong-781.47 \mathrm{~J}, i^{\prime \prime} \cong 258.40 \mathrm{~J}$, correlation coefficient $r^{\prime \prime} \cong-0.9442$, and square mean relative error $13,31 \%$, but we consider these last results as less important than the above-indicated ones, referring to the $E_{\text {imp }}$ and $i_{F}=f\left(t_{\text {heat }}\right)$ dependencies.

\section{Investigations on the Compatibility with the Experimental Data of the Fractal/Multifractal Descriptions of the Fracture Parameters}

Taking into account the errors affecting practically all experimental data, the decision about the compatibility (or incompatibility) of a certain hypothesis (e.g., the fractal character of the fracture surfaces) has to be established using some statistical tests [45-47]. Unfortunately, neither [29] nor [30, 32-38] studied statistically the compatibility of the investigated hypothesis relative to the experimental data, and even these works did not indicate the errors corresponding to the used experimental data.

In order to evaluate the error risk at the rejection of the compatibility of a certain representative point relative to the studied correlation $Y_{i}=f(X)$, it is possible to use both global (for the entire correlation) or local test, respectively. For example, the error risk can be evaluated by means of the expression (see [44-48])

$$
q_{k}=\exp \left\{-\frac{1}{2\left(1-r_{k}^{2}\right)}\left[\left(\frac{Y_{i k}-Y_{i, t k}}{s\left(Y_{i k}\right)}\right)^{2}+\left(\frac{X_{k}-X_{t k}}{s\left(X_{k}\right)}\right)^{2}-2 r_{k}\left(\frac{Y_{i k}-Y_{i, t k}}{s\left(Y_{i k}\right)}\right)\left(\frac{X_{k}-X_{t k}}{s\left(X_{k}\right)}\right)\right]\right\}
$$

where $Y_{i k}$ and $X_{k}$ are the impact energy and the fractal dimension corresponding to the representative point (state) $k(=1,2, \ldots N), Y_{i, t k}$ and $X_{t k}$ are the impact energy and the fractal dimension corresponding to the tangency point of the confidence ellipse centered in $\left(Y_{i k}, X_{k}\right)$ with the studied correlation plot: $Y_{i}=f(X)$, while $r_{k}, s\left(Y_{i k}\right)$, and $s\left(X_{k}\right)$ are the correlation coefficient and the square mean errors corresponding to the individual values $Y_{i k}$ and $X_{k}$. Because these errors are not indicated by the studied work [29], we will try evaluate them from other studies about the fracture energy.

The studies $[31,48]$ of the published works concerning the (multi)fractal correlations of some mechanical (fracture) parameters with the specimen size points out the magnitude orders of the errors corresponding to the fracture energy. The corresponding relative errors are indicated in Table 3. One finds that for concrete specimens, the average relative errors affecting the fracture energy is of (approximately) $7 \%$.

Assuming that the relative errors affecting the values of the fractal dimension are considerably less than those corresponding to the impact energy (approx. 10\%), the expression (7.1) leads to error risks somewhat larger than $2 \%$ associated to the rejection of the compatibility hypothesis of the fractal/multifractal descriptions with the experimental data. It results that the compatibility hypothesis cannot be rejected, but a more sure decision needs imperatively the knowledge of the corresponding measurement errors. 
Table 3: Relative errors corresponding to the experimental data concerning the fracture energy $G_{F}$ for different concrete and rocks specimens.

\begin{tabular}{lcccc}
\hline Material [reference] & Concrete [49] & Dry concrete [50] & Wet concrete [50] & $\begin{array}{c}\text { Red felser } \\
\text { sandstone [50] }\end{array}$ \\
\hline Limits of relative errors & $4.68 \cdots 9.76 \%$ & $4.028 \cdots 16.217 \%$ & $2.502 \cdots 11.585 \%$ & $3.125 \cdots 35.424 \%$ \\
Average relative error & $6.062 \%$ & $8.131 \%$ & $6.473 \%$ & $16.236 \%$ \\
\hline
\end{tabular}

\section{Conclusions}

The accomplished study of the numerical data involved by [29] points out the following main original findings.

(1) The decision concerning the fractal (or multifractal) character of the fracture surfaces of metals needs a previous rigorous study by means of the numerical analysis procedures.

(2) In this aim, both the errors corresponding to the geometrical parameters (perimeters and areas of the slit islands) and to the specific mechanical parameters (impact energies), respectively, are necessary.

(3) Taking into account the considerable differences between the values of the fractal dimension resulting from Figure 1 [29], or indicated in the caption of Figure 1 [29], or in Figure 3 [29], we consider that the correct calculation of the fractal dimension corresponds to the interpretation from work [40], which considers that only the perimeters of the slit islands present a fractal character: $P \propto R^{D-1}$, while the areas of these slit islands present the usual second degree dependence on their radii $A \propto R^{2}$; we have found that this interpretation [40] leads also to an agreement between the data from Figure 1 [29] and the values of the fractal dimension indicated by this work [29].

(4) The accomplished study indicates a multifractal nature of the fracture surfaces of metals, the size distribution of the fractals (involved by this multifractal structure) being also evaluated by this work.

(5) The influence of the temperature of the studied maraging steels heat-treatment on the (a) impact energy to fracture and (b) the fracture surface deformation, measured by its fractal dimensional increment, were also studied, finding the increase of the fracture surface deformation with the heat-treatment temperature, particularly.

(6) Using the evaluated errors affecting the fracture energies of some concrete specimens, we have found that the compatibility hypothesis of the fractal/multifractal descriptions with the experimental data cannot be rejected, but a more sure decision always needs an accurate knowledge of the corresponding measurement errors.

\section{References}

[1] Santa Fe Institute for Studies in the Sciences of Complexity: (i) Bulletin, 2-3 issues/year, 1987-present, (ii) Lectures, 1989-present, (iii) Proceedings, 1986-present.

[2] R. Dobrescu and D. A. Iordache, Complexity Modeling, Politehnica Press, Bucharest, Romania, 2007.

[3] R. Dobrescu and D. A. Iordache, Complexity and Information, Romanian Academy Printing House, Bucharest, Romania, 2010. 
[4] P. W. Anderson, "More is different," Science, vol. 177, no. 4047, pp. 393-396, 1972.

[5] P. W. Anderson, "Physics: the opening to complexity," Proceedings of the National Academy of Sciences of the United States of America, vol. 92, no. 15, pp. 6653-6654, 1995.

[6] K. G. Wilson, "Renormalization group and critical phenomena," Physical Review B, vol. 4, no. 9, pp. 3174-3183, 1971.

[7] I. Prigogine and G. Nicolis, Self-Organization in Non-Equilibrium Systems: From Dissipative Structures to Order through Fluctuations, Wiley, New York, NY, USA, 1977.

[8] S. Solomon and E. Shir, "Complexity; a science at 30," Europhysics News, vol. 34, no. 2, pp. 54-57, 2003.

[9] S. Solomon, "Evolving uniform and nonuniform cellular automata networks," in Annual Reviews of Computational Physics, D. Stauffer, Ed., pp. 243-294, World Scientific, River Edge, NJ, USA, 1995.

[10] A. L. Barabási and R. Albert, "Emergence of scaling in random networks," Science, vol. 286, no. 5439, pp. 509-512, 1999.

[11] R. Albert, H. Jeong, and A. L. Barabási, “Diameter of the world-wide web," Nature, vol. 401, no. 6749, pp. 130-131, 1999.

[12] A. L. Barabási, "Complex networks: from the cell to human diseases," in Proceedings of the International Workshop on Quantitative Biology, Bucharest, Romania, May 2007.

[13] K. Bhattacharya, G. Mukherjee, and S. S. Manna, "The international trade network," in Econo-Physics of Markets and Business Networks, A. Chatterjee and B. K. Chakrabarti, Eds., New Economic Windows Series, p. 139, Springer, New York, NY, USA, 2008.

[14] S. Y. Chen, Wei Huang, and C. Cattani, "Traffic dynamics on complex networks: a survey," Mathematical Problems in Engineering, vol. 2012, Article ID 732698, 23 pages, 2012.

[15] E. G. Backhoum and C. Toma, "Dynamical aspects of macroscopic andquantum transitions due to coherence function and time series events," Mathematical Problems in Engineering, vol. 2010, Article ID 428903, 13 pages, 2010.

[16] E. G. Backhoum and C. Toma, "Specific mathematical aspects of dynamics generated by coherence functions," Mathematical Problems in Engineering, vol. 2011, Article ID 436198, 10 pages, 2011.

[17] C. Cattani and P. Sterian, "Modelling the hyperboloid elastic shell," Scientific Bulletin of University "Politehnica" of Bucharest, Series A, vol. 71, no. 4, pp. 37-44, 2009.

[18] A. A. Gukhman, Introduction to the Theory of Similarity, Academic Press, New York, NY, USA, 1965.

[19] G. I. Barenblatt, Dimensional Analysis, Gordon and Breach, New York, NY, USA, 1987.

[20] G. I. Barenblatt, Scaling, Self-Similarity and Intermediate Asymptotics, Cambridge Texts in Applied Mathematics, Cambridge University Press, Cambridge, UK, 1996.

[21] C. Shannon, "The mathematical theory of communication," Bell System Technical Journal, vol. 27, no. 3, pp. 379-423, 1948.

[22] C. Shannon, "The mathematical theory of communication," Bell System Technical Journal, vol. 27, no. 4, pp. 623-656, 1948.

[23] C. Shannon, "Prediction and entropy of printed English," Bell System Technical Journal, vol. 30, no. 1, pp. 50-64, 1951.

[24] A. Carpinteri and B. Chiaia, "Multifractal nature of concrete fracture surfaces and size effects on nominal fracture energy," Materials and Structures, vol. 28, no. 8, pp. 435-443, 1995.

[25] A. Carpinteri and B. Chiaia, "Multifractal scaling laws in the breaking behaviour of disordered materials," Chaos, Solitons and Fractals, vol. 8, no. 2, pp. 135-150, 1997.

[26] D. A. Iordache, P. P. Delsanto, Şt. Puşcă, and V. Iordache, "Complexity, similitude, and fractals in physics," in Proceedings of the 2nd International Symposium on "Interdisciplinary Applications of Fractal Analysis" (IAFA '05), vol. 3, pp. 7-12, Politehnica Press Publishing House, Bucharest, Romania, May 2005.

[27] P. P. Delsanto, D. A. Iordache, and Şt. Puşcă, "Study of the correlations between different effective fractal dimensions used for fracture parameters descriptions," in Interdisciplinary Applications of Fractal and Chaos, R. Dobrescu and C. Vasilescu, Eds., pp. 136-153, Romanian Academy Printing House, Bucharest, Romania, 2004.

[28] P. P. Delsanto, A. S. Gliozzi, C. L. E. Bruno, N. Pugno, and A. Carpinteri, "Scaling laws and fractality in the framework of a phenomenological approach," Chaos, Solitons and Fractals, vol. 41, no. 5, pp. 2782-2786, 2009.

[29] B. B. Mandelbrot, D. E. Passoja, and A. J. Paullay, "Fractal character of fracture surfaces of metals," Nature, vol. 308, no. 5961, pp. 721-722, 1984.

[30] Y. Ma and T. G. Langdon, "Observations on the use of a fractal model to predict superplastic ductility," Scripta Metallurgica et Materiala, vol. 28, no. 2, pp. 241-246, 1993. 
[31] D. A. Iordache, Şt. Puşcă, G. Toma, V. Păun, A. Sterian, and C. Morarescu, "Analysis of compatibility with experimental data of Fractal descriptions of the fracture parameters," Lecture Notes in Computer Science, vol. 3980, pp. 804-813, 2006.

[32] E. E. Underwood and K. Banerji, "Fractals in fractography," Materials Science and Engineering, vol. 80, no. 1, pp. 1-14, 1986.

[33] C. S. Pande, L. E. Richards, N. Louat, B. D. Dempsey, and A. J. Schwoeble, "Fractal characterization of fractured surfaces," Acta Metallurgica, vol. 35, no. 7, pp. 1633-1637, 1987.

[34] C. W. Lung and Z. Q. Mu, "Fractal dimension measured with perimeter-area relation and toughness of materials," Physical Review B, vol. 38, no. 16, pp. 11781-11784, 1988.

[35] Z. H. Huang, J. F. Tian, and Z. G. Wang, "Study of the slit island analysis as a method for measuring fractal dimension of fractured surface," Scripta Metallurgica et Materialia, vol. 24, no. 6, pp. 962-972, 1990.

[36] R. E. Williford, “Multifractal fracture," Scripta Metallurgica, vol. 22, no. 11, pp. 1749-1754, 1988.

[37] Z.Q. Mu and C. W. Lung, "Studies on the fractal dimension and fracture toughness of steel," Journal of Physics D, vol. 21, no. 5, p. 848, 1988.

[38] H. Su, Y. Zhang, and Z. Yan, Acta Metallurgica Sinica, vol. 3, p. 226, 1990.

[39] Şt. Puşcă, V. Solcan, and D. A. Iordache, "Procedure of extraction of numerical data from experimental plots. Application to the numerical analysis of some fractal studies," in Proceedings of the 5th General Conference of the Balkan Physical Union, pp. 259-264, Vrnjacka Banja, Serbia, August 2003.

[40] J. F. Gouyet, Physique et Structures Fractales, Masson, Paris, France, 1992.

[41] D. L. Davidson, "Fracture surface roughness as a gauge of fracture toughness: aluminium-particulate SiC composites," Journal of Materials Science, vol. 24, no. 2, pp. 681-687, 1989.

[42] K. Levenberg, "A method for the solution of certain problems in least squares," Quarterly of Applied Mathematics, vol. 2, pp. 164-168, 1944.

[43] D. W. Marquardt, "An algorithm fot least-squares estimation of nonlinear parameters," Journal of the Society for Industrial and Applied Mathematics, vol. 11, no. 2, pp. 431-441, 1963.

[44] E. Bodegom, D. W. McClure, P. P. Delsanto et al., Computational Physics Guide, Politehnica Press, Bucharest, Romania, 2009.

[45] W.T. Eadie, D. Drijard, F. E. James, M. Roos, and B. Sadoulet, Statistical Methods in Experimental Physics, North-Holland, Amsterdam, The Netherlands, 1982.

[46] W. Ledermann, Ed., Handbook of Applied Mathematics, vol. 6 of Statistics, John Wiley \& Sons, New York, NY, USA, 1984.

[47] P. W. M. John, Statistical Methods in Engineering and Quality Assurance, John Wiley \& Sons, New York, NY, USA, 1990

[48] D. A. Iordache and V. Iordache, "Compatibility of multi-fractal and similitude descriptions of the fracture parameters relative to the experimental data for concrete specimens," in Proceedings of the 1st South-East European Symposium on Interdisciplinary Approaches in Fractal Analysis, pp. 55-60, Bucharest, Romania, May 2003.

[49] A. Carpinteri and G. Ferro, "Scaling behaviour amd dual renormalization of experimental tensile softening responses," Materials and Structures, vol. 31, no. 5, pp. 303-309, 1998.

[50] M. R. A. van Vliet, Size effects in tensile fracture of concrete and rock, Ph.D. thesis, University of Delft, 2000. 


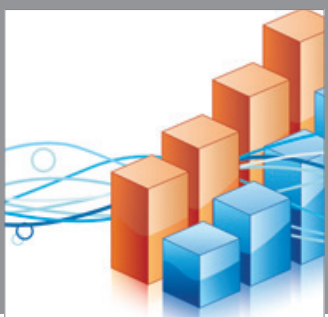

Advances in

Operations Research

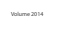

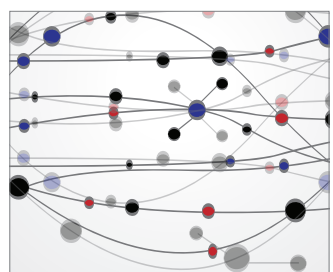

\section{The Scientific} World Journal
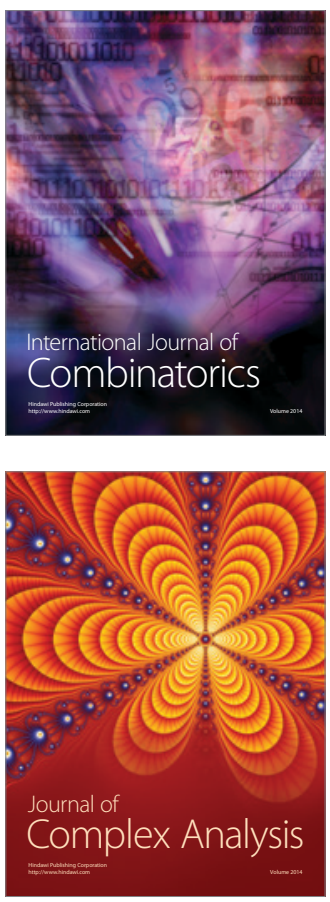

International Journal of

Mathematics and

Mathematical

Sciences
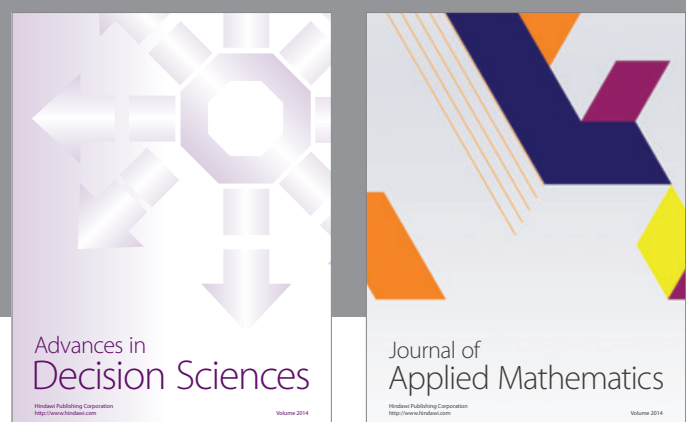

Journal of

Applied Mathematics
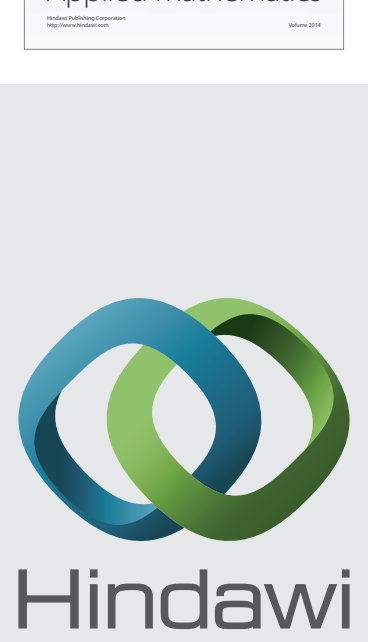

Submit your manuscripts at http://www.hindawi.com
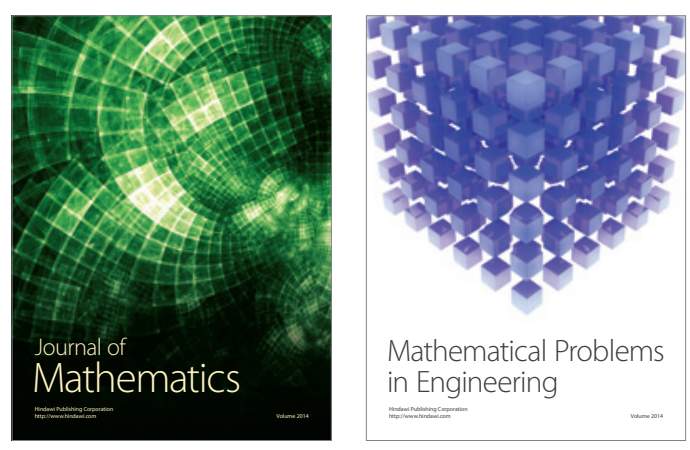

Mathematical Problems in Engineering
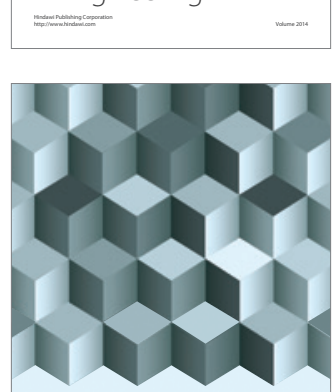

Journal of

Function Spaces
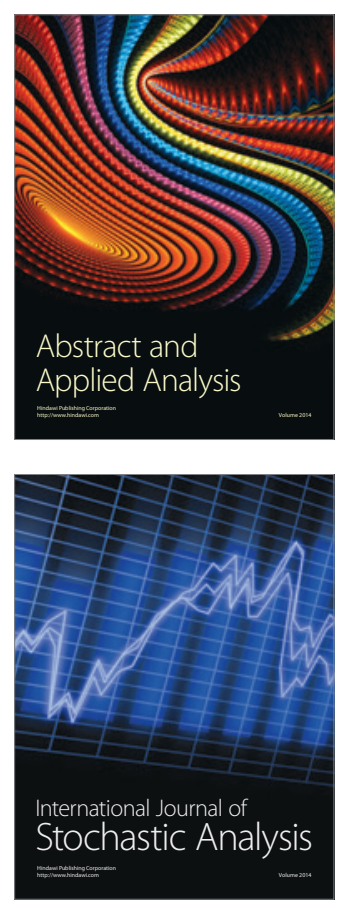

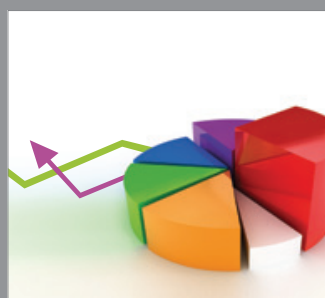

ournal of

Probability and Statistics

Promensencen
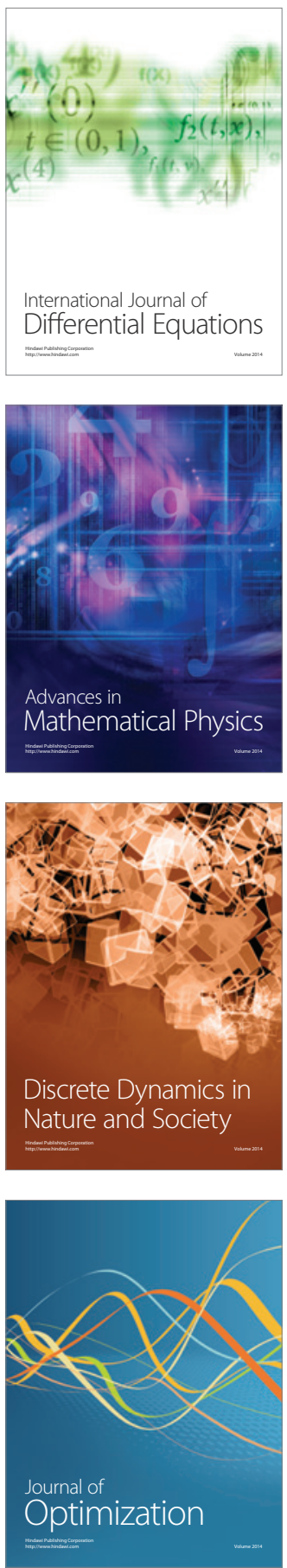